\title{
Pediatric Cytochrome P450 Activity Alterations in Nonalcoholic Steatohepatitis ${ }^{\mathbb{S}}$
}

\author{
Hui Li, Mark J. Canet, John D. Clarke, Dean Billheimer, Stavra A. Xanthakos, Joel E. Lavine, \\ Robert P. Erickson, and Nathan J. Cherrington
}

Department of Pharmacology and Toxicology (H.L., M.J.C., J.D.C., N.J.C.), Department of Epidemiology and Biostatistics (D.B.), and Department of Pediatrics (R.P.E.), University of Arizona, Tucson, Arizona; Cincinnati Children's Hospital, University of Cincinnati, Cincinnati, Ohio (S.A.X.); Columbia University, New York, New York (J.E.L.)

Received July 18, 2017; accepted September 28, 2017

\section{ABSTRACT}

Variable drug responses depend on individual variation in the activity of drug-metabolizing enzymes, including cytochrome P450 enzymes (CYP). As the most common chronic liver disease in children and adults, nonalcoholic steatohepatitis (NASH) has been identified as a source of significant interindividual variation in hepatic drug metabolism. Compared with adults, children present age-related differences in pharmacokinetics and pharmacodynamics. The purpose of this study was to determine the impact of fatty liver disease severity on the activity of a variety of CYP enzymes in children and adolescents. Healthy and nonalcoholic fatty liver disease pediatric subjects aged 12-21 years inclusive received an oral cocktail of four probe drugs: caffeine (CYP1A2, $100 \mathrm{mg}$ ), omeprazole (CYP2C19, $20 \mathrm{mg}$ ), losartan (CYP2C9, $25 \mathrm{mg}$ ), and midazolam (CYP3A4, $2 \mathrm{mg}$ ). Venous blood and urine were collected before administration and 1, 2,4 , and 6 hours after administration. Concentrations of the parent drugs and CYP-specific metabolites were quantified in plasma and urine using liquid chromatography with tandem mass spectrometry. In plasma, the decreased metabolic area under the curve (AUC) ratio, defined as the metabolite AUC to parent AUC, of omeprazole indicated significant decreases of CYP2C19 $(P=0.002)$ enzymatic activities in NASH adolescents, while the urine analyses did not show significant differences and were highly variable. A comparison between the present in vivo pediatric studies and a previous ex vivo study in adults indicates distinct differences in the activities of CYP1A2 and CYP2C9. These data demonstrate that pediatric NASH presents an altered pattern of CYP activity and NASH should be considered as a confounder of drug metabolism for certain CYP enzymes. These differences could lead to future investigations that may reveal unexpected variable drug responses that should be considered in pediatric dosage recommendations.

\section{Introduction}

Nonalcoholic fatty liver disease (NAFLD) encompasses a spectrum of liver disorders ranging from simple steatosis to the progressive form of nonalcoholic steatohepatitis (NASH), which is characterized by inflammation and hepatocyte injury that may lead to liver cirrhosis and even hepatocellular carcinoma (Matteoni et al., 1999; Chalasani et al., 2012). Many studies have demonstrated that the entire spectrum of NAFLD also occurs during childhood (Molleston et al., 2002; Kleiner and Makhlouf, 2016). Concurrent with childhood obesity, NAFLD has developed into a serious concern in pediatric clinical practice and has become a significant health burden in adolescence (AlKhater, 2015). An autopsy-based study estimated the overall prevalence of NAFLD in the American pediatric population at $9.6 \%$, which increased to $17.3 \%$ in adolescents from 15 to 19 years old (Schwimmer et al., 2006).

This work was supported by the National Institutes of Health Eunice Kennedy Shriver National Institute of Child Health and Human Development [Grant R01 HD062489], the National Center for Advancing Translational Sciences [Grant UL1 TR000040], and the National Institute of Environmental Health Science [Grants T32 ES007091 and P30 ES006694].

https://doi.org/10.1124/dmd.117.077644.

S This article has supplemental material available at dmd.aspetjournals.org.
In addition to the direct health concerns from NAFLD or NASH, potential adverse drug reactions (ADRs) associated with this underlying disease have received more and more attention in recent years. NASH has been identified as a potential risk factor for ADRs because profound reprogramming of drug metabolizing enzyme and drug transporter profiles were observed in NASH compared with healthy or simple steatosis populations (Fisher et al., 2009; Hardwick et al., 2011, 2013; Lake et al., 2011). Additionally, in preclinical NASH models, pharmacokinetic alterations were observed for a number of drugs, including ezetimibe, pravastatin, methotrexate, morphine, and metformin (Hardwick et al., 2012, 2014; Clarke et al., 2014, 2015; Dzierlenga et al., 2015). Each of these studies was conducted in adults or animal models reflecting the mature population, which underscores the scarcity of data on the impact of $\mathrm{NASH}$ on drug metabolism and disposition in children. One study by Barshop et al. (2011) reported that after acetaminophen administration, children with NAFLD had statistically significantly higher serum and urinary concentrations of acetaminophen-glucuronide (APAP-gluc), indicating an altered drug metabolism and disposition process along with NAFLD in children. Canet et al. (2015) further demonstrated that regulation of hepatic efflux transporters is altered after the progression to NASH in children, which represents an underlying mechanism for increased acetaminophen-glucuronide in children with NAFLD.

ABBREVIATIONS: ADME, absorption, distribution, metabolism, and excretion; ADR, adverse drug reaction; ALT, alanine aminotransferase; AUC, area under the curve; BMI, body mass index; bp, base pair; CYP, cytochrome P450 enzyme; EXP-3174, losartan carboxylic acid; LC-MS/MS, liquid chromatography with tandem mass spectrometry; NAFLD, nonalcoholic fatty liver disease; NASH, nonalcoholic steatohepatitis; SNP, singlenucleotide polymorphism; UPLC, ultra performance liquid chromatography. 
Furthermore, compared with adults, children experience less certainty regarding drug safety. Physiologic and ontological differences between children and adults result in age-related differences in pharmacokinetics and pharmacodynamics (Anderson, 2010). Therefore, adult data regarding pharmacokinetics and pharmacodynamics cannot simply be extrapolated to children (Kearns et al., 2003). Especially for critically ill children, who are usually exposed to multiple medications, pathologic conditions introduce an additional influence on drug processing (Vet et al., 2011). A systematic review reported $42 \%$ to $100 \%$ of pediatric patients received at least one off-label and/or unlicensed drug (Magalhães et al., 2015), which is associated with the high occurrence of ADRs (Mason et al., 2012).

As the most important drug metabolizing enzyme superfamily, cytochrome p450 enzymes are a major source of variability in drug metabolism. Among 57 putatively functional human CYP enzymes, CYP1A2, CYP2C9, CYP2C19, and CYP3A4 are relatively abundant and are responsible for the biotransformation of approximately $60 \%$ of all clinical drugs (Zanger and Schwab, 2013).

Expression and enzymatic activities of the human CYP enzymes are influenced by multiple factors such as genetic polymorphisms, age, gender, environmental toxicant exposure, dietary constituents, concomitant drug utilization, and disease states (Zanger and Schwab, 2013). Previously, we reported a dramatic alteration of the CYP activity and expression profile in adult NASH patients in an ex vivo study, indicating that disease states such as NASH can alter drug pharmacokinetics by modulating CYP enzymes (Fisher et al., 2009). However, data regarding CYP activity in children with NAFLD are absent.

An accurate characterization of CYP activity is highly practical in predicting the fate of certain classes of drugs in a particular patient or a patient population, which can guide dosing. Compared with an ex vivo study using biopsies, an in vivo approach, which was applied in this study, can yield greater accuracy because it is performed in the intact physiologic context with dynamic blood flow and functioning liver (Hisaka et al., 2010). The purpose of the current study was to determine the in vivo activities of the major CYP isoforms along the spectrum of NAFLD severity in children and adolescents using isoform-specific probe drugs.

\section{Patients and Methods}

Study Design and Population. Twelve pediatric NAFLD patients between the ages of 12 and 21 years inclusive were recruited at Columbia University Medical Center (New York, NY) and Cincinnati Children's Hospital Medical Center (Cincinnati, OH). A screening evaluation based on medical history, physical examination, biochemical screening, and appropriate genotype for one or more of the CYP enzymes under investigation was conducted with potential candidates. The medication history of each subject was examined by screening their medical records, and subjects under long-term or extensive medications that can potentially interfere with CYP activities were excluded from the study.

The NAFLD activity score of each subject was given based on certified pathologists' evaluation of a percutaneous liver biopsy performed previously on each subject for diagnostic standard of care reasons. Briefly, pediatric patients without known allergies to study drugs and with biopsy-confirmed NAFLD with a biopsy performed within 6 months of this study were eligible for inclusion. Seventeen healthy control subjects age 18-21 without any present or past critical liver diseases were recruited at the University of Arizona Medical Center (Tucson, AZ). Subjects with a body mass index (BMI) $<30 \mathrm{~kg} / \mathrm{m}^{2}$ and alanine aminotransferase (ALT) no higher than $26 \mathrm{U} / 1$ for males or $22 \mathrm{U} / \mathrm{l}$ for females were eligible for inclusion in the control group.
Subjects with diabetes mellitus, monogenic inborn errors of metabolism, viral hepatitis, alcohol use, pregnancy, cirrhosis, or other causes of chronic liver disease were excluded from either disease or control group. This study and all study procedures were reviewed and approved by the University of Arizona, Columbia University Medical Center, and Cincinnati Children's institutional review boards before the study commencement. Parents or subjects who met all the eligibility criteria gave written informed consent and informed assent after being given a description of the study before participation in the study.

Saliva was collected from the participating subjects. DNA was isolated from the samples, and genetic analysis of the major relevant variants was performed for polymorphisms in CYP enzymes. The participants were asked to abstain from food and drinks containing caffeine, charbroiled foods, cruciferous vegetables, grapefruit and grapefruit juice, and any medications that can influence CYP activities for 48 hours before and during participation in the study. After an overnight fast, a venous blood sample and a urine sample from each subject were collected immediately before drug administration. Subjects then received the probe drug cocktail orally (100 mg of caffeine, $20 \mathrm{mg}$ of omeprazole, $25 \mathrm{mg}$ of losartan, or $2 \mathrm{mg}$ of midazolam syrup), and postadministration blood and urine collections were obtained at the time points of 1, 2, 4, and 6 hours. Food and drink were allowed after administration with the exception of the previously listed prohibited foods. Blood samples were centrifuged for plasma collection, and the aliquots were stored at $-80^{\circ} \mathrm{C}$.

Chemicals and Reagents. Caffeine citrate (198 mg capsule), losartan potassium (25 mg tablet), omeprazole (20 $\mathrm{mg}$ capsule), and midazolam $\mathrm{HCl}(2 \mathrm{mg} / \mathrm{ml})$ were purchased through Reed's Compounding Pharmacy (Tucson, AZ). Caffeine, losartan, omeprazole, midazolam, 1'-hydroxymidazolam, and phenacetin standards for liquid chromatography with tandem mass spectrometry (LC-MS/MS) analysis were purchased from Sigma-Aldrich (St. Louis, MO). Paraxanthine, paraxanthine-D3, losartan carboxylic acid (E3174), and 5-hydroxyomeprazole were purchased from Toronto Research Chemicals (Toronto, ON, Canada). All other chemicals were obtained from commercial sources.

Cytochrome P450 Genotyping. CYP2C9*2 (restriction enzyme analysis with AvaII on a 241 base pair (bp) polymerase chain reaction fragment flanking the single-nucleotide polymorphism [SNP]), CYP2C9*3 (Sanger sequencing of a 259-bp fragment flanking the SNP), CYP2C19*2 (restriction enzyme analysis with BstNI of a 201-bp fragment flanking the SNP), CYP2C19*17 (Sanger sequencing of a 201-bp fragment flanking the SNP), CYP3A4*1b (Sanger sequencing of a 216-bp fragment flanking the SNP), and CYP3A5*3 (Sanger sequencing of a 182-bp fragment flanking the SNP) typings were performed on all participants. CYP3A5*6 typing (restriction enzyme digestion with DdeI of a 127-bp fragment flanking the SNP) was performed on all Hispanics, CYP3A5*7 typing (Sanger sequencing of a 227-bp fragment flanking the SNP) was performed on all African Americans, and CYP2C19*3 typing (restriction enzyme analysis with BamHI of a 224-bp fragment flanking the SNP) were performed on all Asian or Pacific Islanders. Cytochrome P450 genotyping primer sequences for the polymerase chain reaction fragment amplifications are available on request.

Laboratory Analysis. An ALT assay kit was used to perform the ALT quantification in the baseline plasma samples according to the manufacturer's protocol (Cayman Chemical, Ann Arbor, MI). A colorimetric creatinine assay was used to quantify the creatinine in the baseline urine of each participant (R\&D Systems, Minneapolis, MN).

Sample Preparation and LC-MS/MS Method. Plasma samples $(400 \mu \mathrm{l})$ were spiked with an internal standard $(10 \mu \mathrm{l}$ of $1000 \mathrm{ng} / \mathrm{ml}$ phenacetin), and were diluted by $1200 \mu \mathrm{l}$ of high-performance liquid chromatography grade water. We loaded $1600 \mu \mathrm{l}$ of the diluted sample 
into an Evolute ABN SPE (solid-phase extraction) cartridge (30 mg; Biotage, Uppsala, Sweden), which was previously conditioned with $1 \mathrm{ml}$ of methanol and equilibrated with $1 \mathrm{ml}$ of water, and was washed with $1 \mathrm{ml}$ of $5 \%$ methanol twice and then eluted with $500 \mu \mathrm{l}$ of methanol. The eluent was evaporated to dryness at room temperature under a stream of nitrogen, reconstituted in $100 \mu \mathrm{l}$ of $40 \%$ acetonitrile, and a volume of $10 \mu \mathrm{l}$ was injected into the chromatographic system for separation and analysis (Supplemental Fig. 1).

The urine sample was 1:4 diluted with high-performance liquid chromatography water; $400 \mu \mathrm{l}$ of diluted urine was spiked with an internal standard $(10 \mu \mathrm{l}$ of phenacetin $(1000 \mathrm{ng} / \mathrm{ml})$ and $10 \mu \mathrm{l}$ of paraxanthine-d3 $(4000 \mathrm{ng} / \mathrm{ml}))$. The spiked urine was processed with an Evolute ABN SPE cartridge after the same procedure of plasma sample processing (Supplemental Fig. 2).

Analysis was performed with a Waters (Milford, MA) Micromass Quattro Premier XE tandem mass spectrometer coupled to an Acquity ultra performance liquid chromatography (UPLC) in the Arizona Laboratory for Emerging Contaminants at the University of Arizona. The analytes were separated using $0.1 \%$ formic acid in water (A) and $0.1 \%$ formic acid in acetonitrile (B) as mobile phases at a flow rate of $0.125 \mathrm{ml} / \mathrm{min}$ through a Waters Acquity UPLC BEH C18 column $(1.7 \mu \mathrm{m}, 2.1 \times 50 \mathrm{~mm})$. The UPLC gradient started at $40 \% \mathrm{~B}$ and increased to $50 \%$ B over 1.83 minutes, sequentially increased to $100 \%$ $\mathrm{B}$ at 1.88 minutes and continued for 0.9 minutes, then was equilibrated to $40 \%$ B for 1 minute before the next injection. Data acquisition was performed in multiple reactions monitoring mode with positive electrospray positive ionization. The mass transitions were as follows: $\mathrm{m} / \mathrm{z} 195 \rightarrow 138$ for caffeine, $\mathrm{m} / \mathrm{z}, 181 \rightarrow 124$ for paraxanthine, $\mathrm{m} / \mathrm{z}$ $346 \rightarrow 198$ omeprazole, $\mathrm{m} / \mathrm{z} \quad 362 \rightarrow 214$ for 5 -hydroxyomeprazole, $\mathrm{m} / \mathrm{z}$ $326 \rightarrow 291$ for midazolam, $\mathrm{m} / \mathrm{z} \quad 342 \rightarrow 324$ for 1-hydroxymidazolam, $m / z, 423 \rightarrow 207$ for losartan, $\mathrm{m} / \mathrm{z} 437 \rightarrow 207$ for EXP-3174 (losartan carboxylic acid), $m / z, 180 \rightarrow 110$ for phenacetin, and $m / z, 184 \rightarrow 127$ for paraxanthine-D3 (Supplemental Table 1).

Variance Comparisons and Statistical Analyses. Data used for plotting pharmacokinetic curves are represented as mean \pm S.E.M. The pharmacokinetics area under the curve (AUC) of the parent drug or metabolite was computed for each participant. The AUC ratio, defined as the metabolite AUC divided by the parent AUC, was computed, and the box and whisker plots of AUC ratio generated represent the median and the minimum and maximum. Comparisons among the normal, steatosis, and NASH groups were made with one-way analysis of variance Bonferroni's multiple comparison test, and $P<0.05$ indicated a statistically significant difference from control. All analyses were performed using GraphPad Prism software (GraphPad Software, La Jolla, CA).

Enzymatic activity data collected from this study were compared with results from a previous ex vivo study conducted with adult NASH patients (Fisher et al., 2009). The relative CYP enzymatic activity of each NASH participant was computed by normalizing to the mean CYP activity of the normal group. For comparison, the mean difference of CYP activity comparing the steatosis or NASH groups to the control group was computed from a linear model with logarithmic transformed relative activity.

\section{Results}

Clinical Characteristics of the Study Population. The clinical characteristics of study populations are summarized in Table 1. Seventeen healthy subjects were categorized as normal. According to the steatohepatitis diagnosis by liver biopsy, seven subjects were categorized as steatosis, and five were confirmed as NASH patients. The mean age and BMI were $19.3 \pm 0.3$ years and $23.8 \pm 1.2 \mathrm{~kg} / \mathrm{m}^{2}$,
TABLE 1

Baseline characteristics of the study population

Data are presented as mean (S.D.) unless indicated as $n(\%)$. BMI was calculated as weight in kilograms divided by height in meters squared. NAFLD activity score was assessed on a scale of $0-8$, with higher scores showing more severe disease; the components of this measure were steatosis (assessed on a scale of 0-3), lobular inflammation (assessed on a scale of 0-3), and hepatocellular ballooning (assessed on a scale of 0-2). Portal inflammation was assessed on a scale of $0-2$, with higher scores showing more severe inflammation. Mean fibrosis stage was assessed on a scale of $0-4$, with higher scores showing more severe fibrosis.

\begin{tabular}{lccc}
\hline \multicolumn{1}{c}{ Characteristics } & Healthy & Steatosis & NASH \\
\hline Age, y & $19.3(1.2)$ & $15.3(2.3)$ & $17.4(1.7)$ \\
Female sex, $n(\%)$ & $8(47.1)$ & $5(71.4)$ & $2(40)$ \\
Hispanic ethnicity, $n(\%)$ & $3(17.6)$ & $7(100)$ & $5(100)$ \\
Race, $n(\%)$ & & & \\
Asian or Pacific Islander & $5(29.4)$ & 0 & 0 \\
Caucasian & $10(58.8)$ & 0 & 0 \\
$\geq 2$ Races & 0 & $1(14.3)$ & 0 \\
Height, cm & $170.3(11.0)$ & $163.5(10.2)$ & $166.5(11.6)$ \\
Weight, kg & $69.3(15.4)$ & $89.6(24.4)$ & $107.2(22.5)$ \\
BMI, kg/m ${ }^{2}$ & $23.8(4.8)$ & $33.1(5.3)$ & $38.3(3.3)$ \\
ALT, U/L & $18(6)$ & $79(70)$ & $73(37)$ \\
Liver histology & & & \\
NAFLD activity score (NAS) & NA & $4.0(1.4)$ & $5.6(0.5)$ \\
Steatosis score & NA & $2.1(0.7)$ & $2.2(0.8)$ \\
Lobular inflammation score & NA & $1.4(0.8)$ & $2.0(0.0)$ \\
Portal inflammation score & NA & $0.6(0.5)$ & $0.6(0.5)$ \\
Hepatocellular ballooning score & NA & $0.4(0.5)$ & $1.6(0.9)$ \\
Fibrosis stage & NA & $1(1.7)$ & $2(2.3)$ \\
CYP polymorphisms, $n(\%)$ & & & \\
CYP2C9*2 & $4(23.5)$ & 0 & $1(20)$ \\
CYP2C9*3 & $4(23.5)$ & $1(14.3)$ & 0 \\
CYP2C19*2 & $4(23.5)$ & $1(14.3)$ & 0 \\
CYP2C19*17 & $4(23.5)$ & 0 & $1(20)$ \\
CYP3A4*1b & $3(17.6)$ & $3(42.9)$ & $1(20)$ \\
CYP3A5*3 & $5(29.4)$ & $4(57.1)$ & $1(20)$ \\
\hline
\end{tabular}

NA, not applicable.

respectively, for the healthy population; $15.3 \pm 0.9$ years and $33.1 \pm$ $2.0 \mathrm{~kg} / \mathrm{m}^{2}$, respectively, for the steatosis group; and $17.4 \pm 0.7$ years and $38.3 \pm 1.5 \mathrm{~kg} / \mathrm{m}^{2}$, respectively, for the NASH group. Females composed $47.1 \%$ of the controls, $71.4 \%$ of patients with steatosis and $40 \%$ of the NASH population. The control group had a diverse ethnic background, but $100 \%$ of the steatosis and NASH subjects were of Hispanic ethnicity.

Compared with the control group $(17.7 \pm 1.5 \mathrm{U} / \mathrm{l})$, the NAFLD patients had a statistically significantly higher ALT level $(79.3 \pm 26.3$ for steatosis and $72.5 \pm 16.5$ for NASH). The average NAFLD activity score was $4.0 \pm 0.5$ for steatosis and $5.6 \pm 0.2$ for NASH. All NAFLD (steatosis and NASH) patients showed significant steatosis with an average score of 2, indicating 34\%-66\% fatty infiltration of hepatocytes with macrovesicular steatosis was evident. The NASH patients had significantly higher scores of lobular inflammation and hepatocellular ballooning than simple steatosis.

Polymorphisms of CYP2C9*2, CYP2C9*3, CYP2C19*2, CYP2C19*17, CYP3A4*1b, and CYP3A $5 * 3$ were characterized in all subjects in this study. CYP3A5*6 typing was performed on all Hispanics, CYP3A5*7 typing was performed on all African Americans, and CYP2C19*3 typing was performed on all Asian or Pacific Islander participants. The number of polymorphic subjects of each CYP isoform is reported in Table 1.

Pharmacokinetics of Probe Drugs in Plasma. The pharmacokinetic curves of probe drugs (caffeine, losartan, omeprazole, and midazolam) and their metabolites (paraxanthine, EXP-3174, 5-hydroxyomeprazole, and 1-hydroxymidazolam) in plasma are shown in Fig. 1A. The metabolic AUC ratio of each probe drug was used to represent the enzymatic activity of each respective CYP isoform. The metabolic AUC ratio of omeprazole was decreased by $71 \%$ in NASH 
A

Caffeine

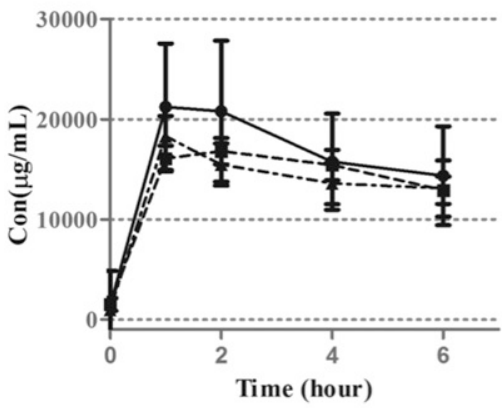

Losartan

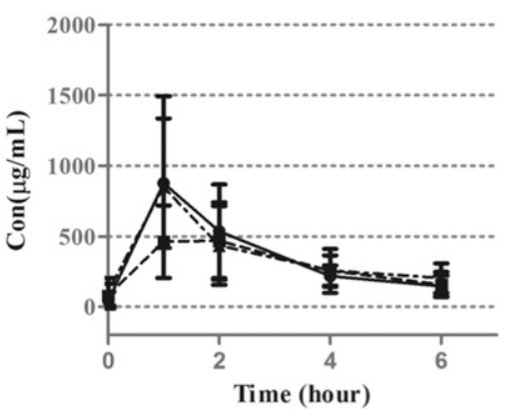

Omeprazole

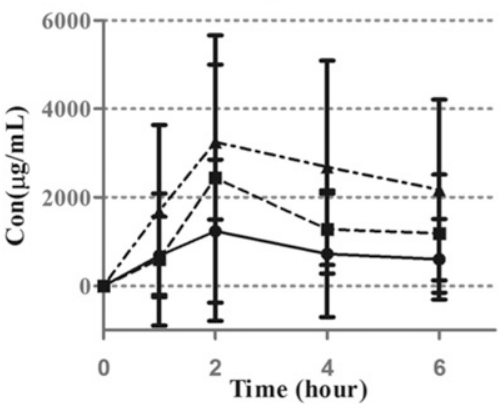

Midazolam

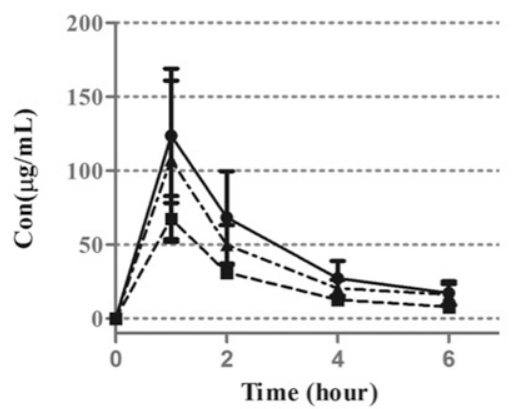

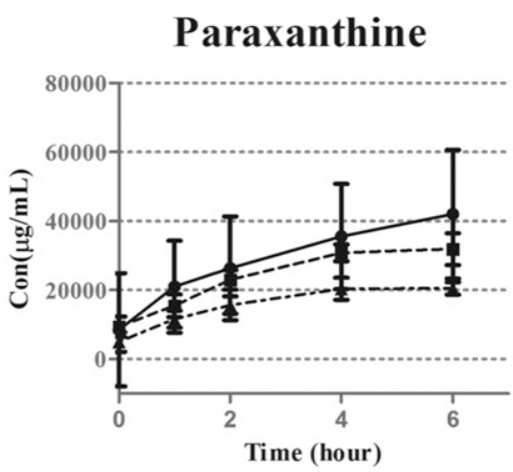

EXP3174

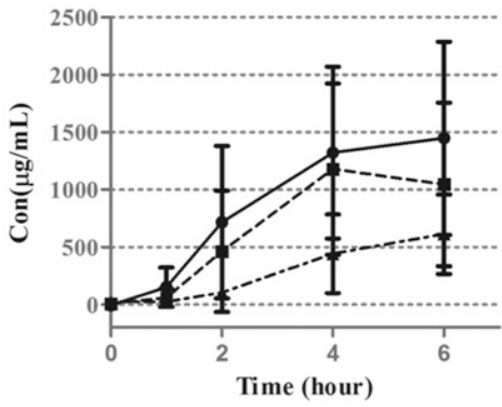

50H-Omeprazole

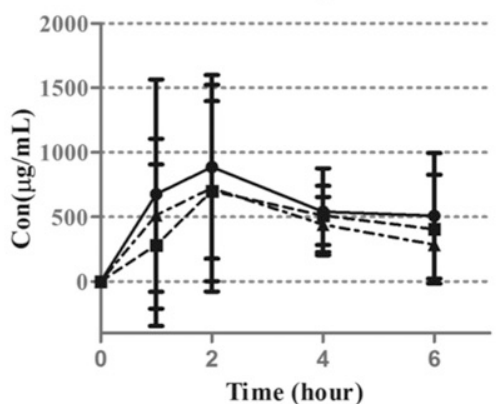

10H-Midazolam

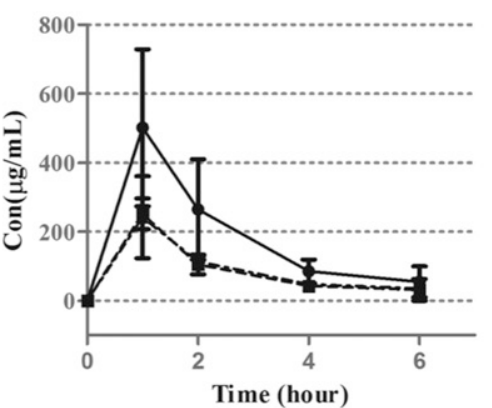

B CYP1A2

- Normal

ㅍ. Steatosis

-A- NASH

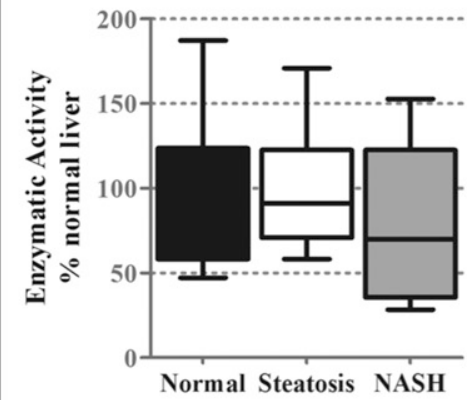

CYP2C9

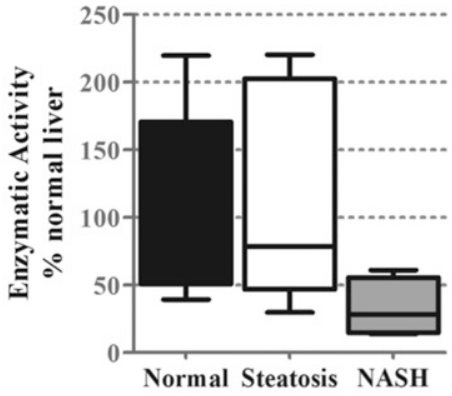

CYP2C19

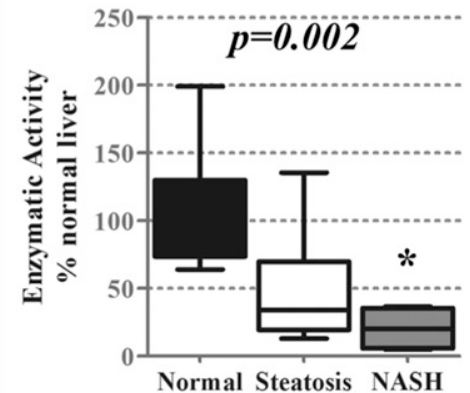

CYP3A4

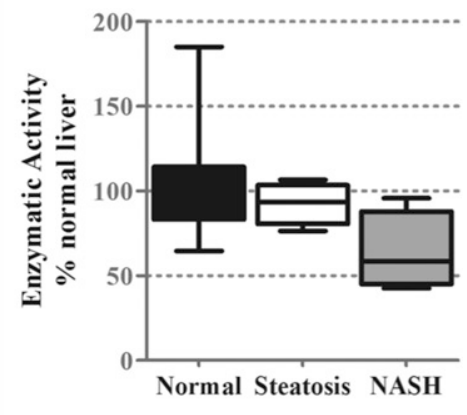

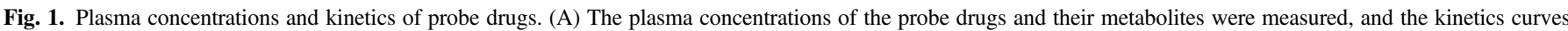

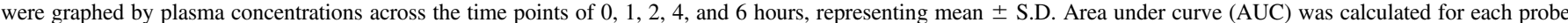

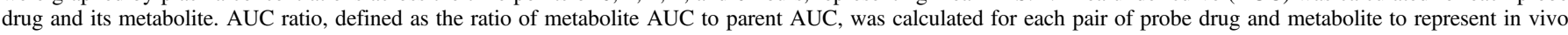

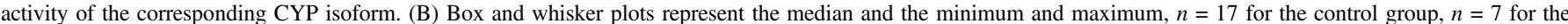

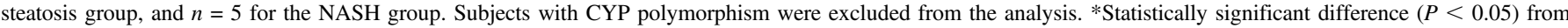

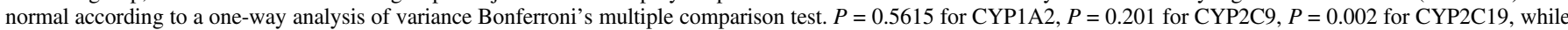
the change in the NASH group was significant compared with normal, according to post test, and $P=0.084$ for CYP3A4 
patients compared with healthy subjects, indicating a significant loss of CYP2C19 function in NASH adolescents, although there was no significant difference between the steatosis and NASH groups. Even though the pharmacokinetic curves of caffeine, losartan, and midazolam as well as their metabolites were differentiated among the normal, steatosis, and NASH groups, calculation of metabolic AUC ratios offset these changes, suggesting that the enzymatic activity of CYP1A2, CYP2C9, and CYP3A4 was not significantly altered in adolescents with steatosis or NASH. Polymorphic subjects were excluded from the pharmacokinetic data analysis of the corresponding probe drug; the values of the AUC ratio of polymorphic subjects are summarized in Tables 2, 3, and 4 .

Pharmacokinetics of Probe Drugs in Urine. Direct urinary concentration was normalized to urinary creatinine concentration (one healthy subject urine sample was absent, $n=16$ ). The pharmacokinetic curves were graphed using normalized urinary concentrations (Fig. 2A). Compared with the plasma data, the urine data were more variable due to the inconsistency of urine samples. Statistical significance for altered metabolic AUC ratios of probe drugs was not achieved in the urine analysis; however, the decreasing trend in the metabolic AUC ratio of omeprazole was consistent with the plasma data and suggested a decrease in CYP2C19 activity. As with the plasma analysis, polymorphic subjects were excluded from the data analysis, and the values of polymorphic subjects are reported in Tables 2, 3, and 4.

Variance Comparison between Adolescent and Adult Studies. A variance comparison of CYP activities was performed between the present pediatric study and a previous study in adults (Fisher et al., 2009). Relative enzymatic activities of steatosis and NASH subjects from both studies are presented in logarithmic scale in Fig. 3. CYP1A2 activity was significantly decreased, and CYP2C9 activity was increased in adults with NASH, which was not shown in adolescents. In adults, CYP2C19 activity was significantly decreased in both steatosis and NASH, while a significant decrease of CYP2C19 activity was seen only in adolescents with NASH.

\section{Discussion}

Although pediatric NAFLD shares similarities with adult NAFLD with respect to a close association with obesity and cardiometabolic risk factors, there are known differences in some histologic features as well as environmental toxins and pubertal hormonal exposures that may influence the natural history and pathogenesis at younger ages (Crespo et al., 2016). Therefore, adult data for drug metabolism and disposition alterations in NAFLD and NASH cannot simply be extrapolated to children. This study attempted to characterize the in vivo CYP activities in children with NAFLD to precisely reflect the impact of NAFLD on CYP-associated drug metabolism in the pediatric population.

TABLE 2

Metabolic AUC ratio of losartan in CYP2C9 polymorphic subjects Data are presented as mean (S.D., $n$ ).

\begin{tabular}{clll}
\hline Group & \multicolumn{1}{c}{ Genotype } & Plasma AUC Ratio & Urine AUC Ratio \\
\hline \multirow{2}{*}{ Control } & Reference Sequence(CC/AA) & $2.45(1.73,9)$ & $0.58(0.25,8)$ \\
& CYP2C9*2(CT/AA) & $1.23(0.56,4)$ & $0.51(0.21,4)$ \\
& CYP2C9*3(CC/AC) & $1.53(0.84,4)$ & $0.67(0.27,4)$ \\
\multirow{2}{*}{ Steatosis } & Reference Sequence(CC/AA) & $2.68(1.94,6)$ & $0.53(0.11,6)$ \\
& CYP2C9*3(CC/AC) & $0.19(\mathrm{NA}, 1)$ & $0.13(\mathrm{NA}, 1)$ \\
NASH & Reference Sequence(CC/AA) & $0.80(0.53,4)$ & $0.54(0.14,4)$ \\
& CYP2C9*2(CT/AA) & $2.06(\mathrm{NA}, 1)$ & $0.35(\mathrm{NA}, 1)$ \\
\hline
\end{tabular}

NA, not applicable.
Pediatric subjects are categorized by the U.S. Food and Drug Association as adolescents between the ages of 12 and 21. The University of Arizona Institutional Review Board required that control subjects for this study be in the age range of 18 to 21 years. Age-related changes of CYP isoforms have been extensively studied in this age range, and the most dramatic changes are observed in earlier stages of infanthood to early childhood (Kearns et al., 2003; Salem et al., 2013). Additionally, because most of the current study subjects had reached puberty, the minor inequality of ages between the groups was not likely to introduce significant variability in CYP activity.

The occurrence of NAFLD in children involves a complex interaction of genetic and environmental contributing factors (Loomba et al., 2009). A strong association between NAFLD in children and childhood obesity is well established (Schwimmer et al., 2003). In this study, a BMI of $<30 \mathrm{~kg} / \mathrm{m}^{2}$ was applied as an inclusion criteria for the control group, which excluded subjects with obesity from this group. As expected, patients with histologically confirmed NAFLD had a significantly higher BMI.

Ethnicity is another traditional risk factor of NASH with a high prevalence of NASH in patients with Hispanic origin (Bambha et al., 2012), which was reflected in the ethnic composition of the NASH group in the study. A significant increase of ALT was observed in steatosis $(79.3 \pm 26.3 \mathrm{U} / \mathrm{l})$ and the NASH group $(72.5 \pm 16.5 \mathrm{U} / \mathrm{l})$ compared with the control group $(19.1 \pm 2.8 \mathrm{U} / \mathrm{l})$, which is consistent with previously reported elevated ALT in pediatric NAFLD (Molleston et al., 2014).

Heritable genetic variations in CYP enzymes can significantly alter function of these enzymes. To minimize this variability, genotyping of high-frequency SNPs was performed for all subjects. CYP2C9*2 and CYP2C9*3 are two most frequent and well investigated SNPs of CYP2C9, both of which lead to reduced CYP2C9 activity (Kidd et al., 2001; Rettie and Jones, 2005). CYP2C19*2 is a high-frequency allele in Caucasians, leading to reduced function of CYP2C19 and a decrease in the elimination of omeprazole (Zhou et al., 1999), while CYP2C19*17 is a gain-of-function allele leading to increased activity of CYP2C19 (Zabalza et al., 2012). CYP3A4*1b occurs in the Caucasian population at $2 \%-9 \%$, though the effect on CYP3A4 activity is controversial (Zanger and Schwab, 2013). Polymorphic subjects with a specific SNP were excluded from the data analysis of the corresponding CYP isoform, though the AUC ratio values of these subjects are reported separately in Tables 2-4. In addition, the majority of racial/ethnic differences in drug metabolism have been attributed to genetic polymorphisms (McGraw and Waller, 2012; Zanger and Schwab, 2013). Exclusion of polymorphic individuals in the current study design limits any potential racial/ethnic influence.

Alterations of metabolizing enzyme activities have been observed in critical diseases as well as compromised conditions in children. NAFLD is a complex disease that can be attributed to a variety of factors, including central obesity, diabetes mellitus, and dyslipidemia (Schwimmer et al., 2003; Newton et al., 2016). The specific impact of obesity on drug metabolism and elimination has been investigated intensively. To summarize these studies, clearance mediated by CYP1A2, CYP2C9, CYP2C19, and CYP2D6 tended to be higher in obese individuals, indicating an increase of metabolic activity of these CYP isoforms, although most studies were conducted in vivo in adults without statistically significance (Brill et al., 2012). In contrast, in an in vivo evaluation of CYP1A2 activity in children with obesity aged between 6 and 10 years using the urinary metabolism ratio of caffeine, a nonsignificant lower CYP1 A2 enzymatic activity was reported (Chiney et al., 2011). Additionally, in people with obesity, multiple known CYP3A4 substrates demonstrated a decreased metabolism and clearance in vivo, with some achieving statistical significance, indicating a trend 
TABLE 3

Metabolic AUC ratio of omeprazole in CYP2C19 polymorphic subjects

Data are presented as mean (S.D., $n$ ).

\begin{tabular}{clcc}
\hline Group & \multicolumn{1}{c}{ Genotype } & Plasma AUC Ratio & Urine AUC Ratio \\
\hline \multirow{2}{*}{ Control } & Reference Sequence(GG/CC) & $1.24(0.46,10)$ & $51.00(55.19,10)$ \\
& CYP2C19*2(GA/CC) & $0.63(0.20,3)$ & $8.56(9.25,3)$ \\
& CYP2C19*17(GG/CT) & $0.54(0.47,2)$ & $43.54(38.39,2)$ \\
& CYP2C19*17(GG/TT) & $0.87(\mathrm{NA}, 1)$ & $17.14(\mathrm{NA}, 1)$ \\
Steatosis & CYP2C19*2\&CYP2C19*17(GA/CT) & $1.64(\mathrm{NA}, 1)$ & $26.18(\mathrm{NA}, 1)$ \\
& Reference Sequence(GG/CC) & $0.55(0.51,5)$ & $38.05(37.71,6)$ \\
NASH & CYP2C19*2(GA/CC) & $0.49(\mathrm{~N} / \mathrm{A}, 1)$ & $49.46(\mathrm{NA}, 1)$ \\
& Reference Sequence(GG/CC) & $0.24(0.19,4)$ & $17.51(17.02,4)$ \\
& CYP2C19*17(GG/CT) & $1.97(\mathrm{NA}, 1)$ & $23.45(\mathrm{NA}, 1)$ \\
\hline
\end{tabular}

NA, not applicable.

toward lower CYP3A4 metabolic activity in obese individuals (Brill et al., 2012).

A critical component of NASH, inflammation has also been recognized as an important factor in altering drug metabolism. Reduced activity of CYP3A, CYP1A2, CYP2C9, CYP2C19, and CYP2D6 were reported in patients with an acute infection or inflammatory disease, and further mechanistic studies in animals and human hepatocytes showed that both lipopolysaccharide exposure and the presence of elevated cytokines (interleukin-1, interleukin-6, tumor necrosis factor- $\alpha$, interferon- $\gamma$ and transforming growth factor- $\beta$ ) could lead to downregulation of CYP activity (Morgan, 1997; Aitken and Morgan, 2007; Yang et al., 2010).

In addition, inflammation has been shown to affect the expression and activity of important drug transporters (Cressman et al., 2012). Interestingly, inflammation has been recognized as a major driving force in the progression from simple steatosis to steatohepatitis (Stojsavljević et al., 2014). In a previous global gene expression study, the vast majority of altered absorption, distribution, metabolism, and excretion (ADME) genes were observed in NASH rather than in simple steatosis, suggesting that ADME reprograming in NAFLD could be a simultaneous or sequential event of the second-hit in NASH progression associated with the inflammatory response (Lake et al., 2011).

In the current study, a significant decrease of CYP2C19 activity was observed in adolescents with NASH but not steatosis, and no significant changes were found in CYP1A2, CYP2C9, or CYP3A4 (Figs. 1 and 2), which is partially consistent with data from other inflammatory conditions but very different from observations in populations with obesity (Brill et al., 2012). Although the significance was only observed in plasma samples but not urine samples, this may be due to variability in urine data obtained from the analysis.

In adults, the impact of NASH on ADME pathways has been well established (Fisher et al., 2009; Hardwick et al., 2010, 2011, 2013). In a previous ex vivo study in adult NASH patients, diverse patterns of CYP alterations were observed (Fisher et al., 2009). For CYP1A2, a significant decrease in ex vivo activity occurred with a decreasing trend of mRNA expression as well as a significant decrease of protein expression. CYP2C9 activity was significantly increased, though neither mRNA nor protein expression was changed, indicating a posttranslational mechanism of regulation. However, changes in CYP1A2 and CYP2C9 activity were not found in either steatosis or NASH adolescents. A significant decrease in CYP2C19 activity was observed in both NASH adults and adolescents. Although there was some decrease in adult CYP3A4 activity without statistical significance, the CYP3A4 activity in adolescents was not significantly changed. In summary, the distinct pattern of CYP activity alterations in adolescents with NASH compared with adults suggests the possibility of ontogenetic factors in the response to inflammatory disease for these CYP isoforms.

Although pharmacokinetic studies have demonstrated age-related differences in very young patients for some CYP activities, our present study demonstrates differences in response to disease in patients as old as 12-21 years, the age at which the activity of all CYP isoforms investigated was thought to be similar to adults (Hines, 2007). It is noteworthy that the ex vivo study was performed with human liver microsomes while the CYP activities in this pediatric study were evaluated in an intact physiologic condition. Therefore, the differences found between the two studies may reflect the differences in CYP activities in the different reaction environments.

CYP-mediated drug metabolism in adolescents with NASH can decrease up to $80 \%$, which creates the potential for an increased incidence of variable drug responses. Particularly for those drugs with severe side effects, a dramatic decrease of drug metabolism and elimination can have significant implications on the therapeutic use of a drug and can increase the risk of ADRs or drug toxicities. Furthermore, in addition to CYP enzymes, alterations in other drug metabolizing enzymes and important drug transporters have been demonstrated in adult NASH patients (Hardwick et al., 2010, 2011, 2013) as well as pediatric NASH patients (Canet et al., 2015). In other words, patients with progressive NASH may have a profoundly different context regarding drug biotransformation and disposition. This makes the prediction of pharmacokinetics of drugs in this population very difficult, and may have an implication on clinical drug trials.

Though data on other ADME genes in pediatric patients with NASH are absent, additional differences between children and adults could be expected. Therefore, future research regarding the unique response of children to NASH and in particular more pharmacokinetic studies of high-risk drugs are warranted in pediatric NASH patients. Physiologically based pharmacokinetic modeling may be particularly useful in translating the current findings into potential clinical implications and facilitating estimates for appropriate dose adjustments.

TABLE 4

Metabolic AUC ratio of midazolam in CYP3A4 polymorphic subjects

Data are presented as mean (S.D., $n$ )

\begin{tabular}{llll}
\hline & \multicolumn{1}{c}{ Genotype } & Plasma AUC Ratio & \multicolumn{1}{c}{ Urine AUC Ratio } \\
\hline \multirow{2}{*}{ Control } & Reference Sequence(AA) & $3.84(1.15,14)$ & $20.48(10.66,14)$ \\
& CYP3A4*1b(AG) & $3.36(0.71,2)$ & $10.24(\mathrm{NA}, 1)$ \\
Steatosis & Reference Sequence(AA) & $3.56(0.48,4)$ & $16.97(11.35,4)$ \\
& CYP3A4*1b(AG) & $2.46(0.41,3)$ & $24.90(5.54,3)$ \\
NASH & Reference Sequence(AA) & $2.45(0.89,4)$ & $20.61(8.85,4)$ \\
& CYP3A4*1b(AG) & $1.63(\mathrm{NA}, 1)$ & $15.22(\mathrm{NA}, 1)$ \\
\hline
\end{tabular}

NA, not applicable. 
A

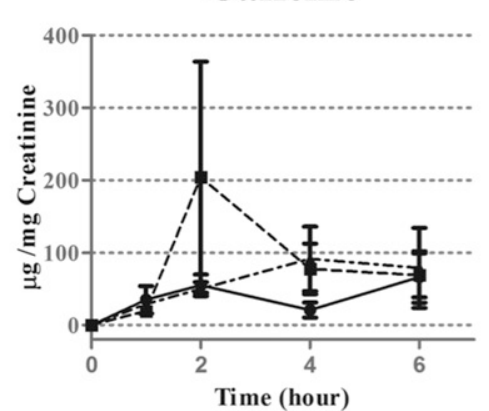

Losartan

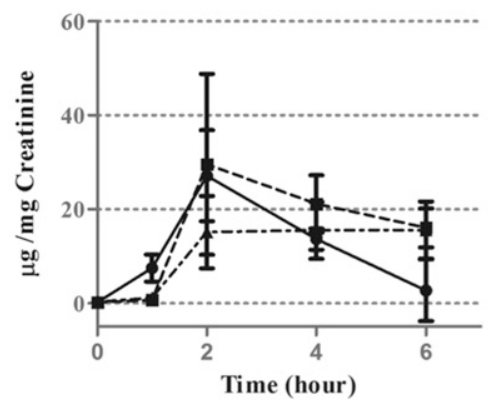

Omeprazole

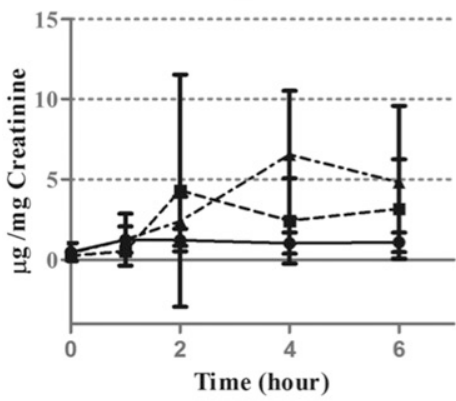

Midazolam

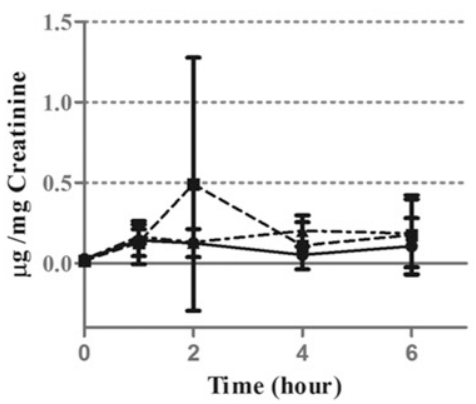

Paraxanthine

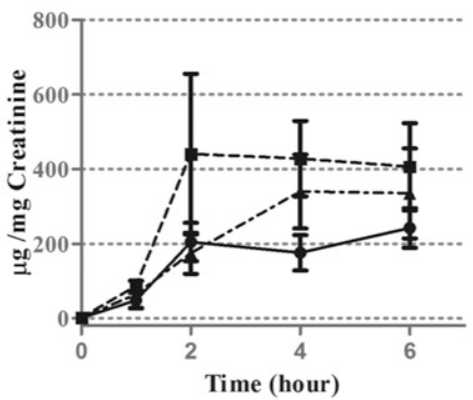

EXP3174

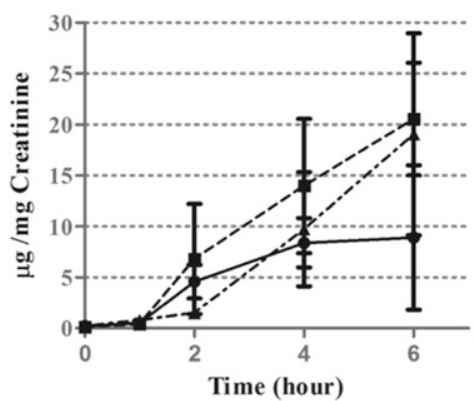

5OH-Omeprazole

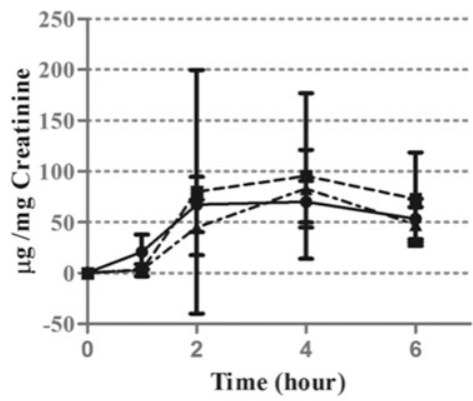

10H-Midazolam

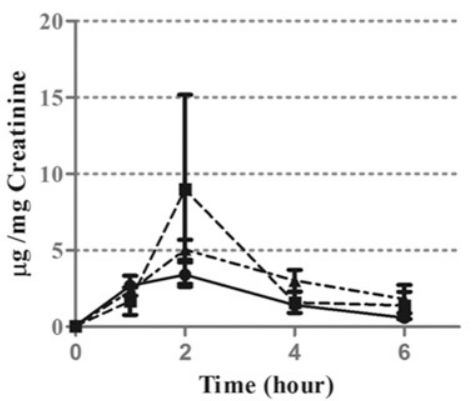

B

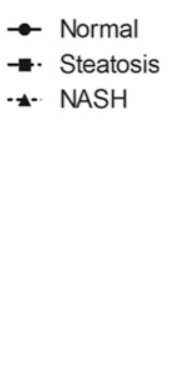

$\rightarrow$ Steatosis

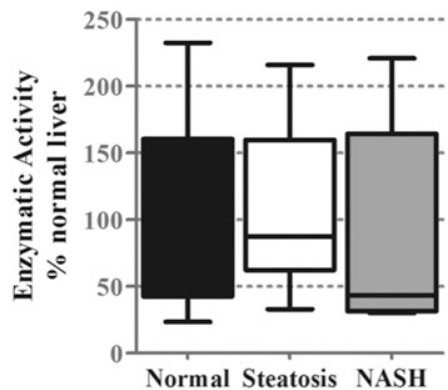

CYP2C9

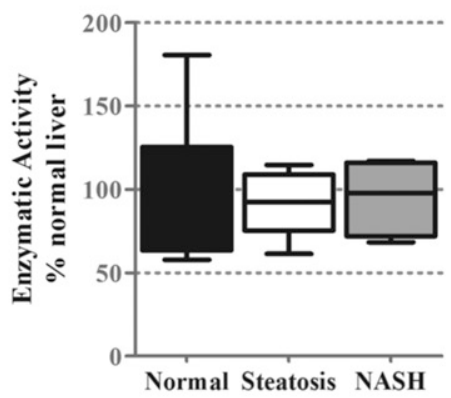

CYP2C19

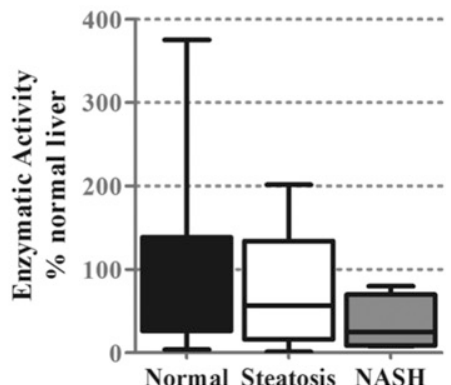

CYP3A4

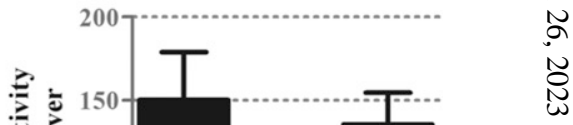

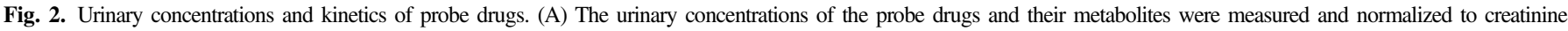

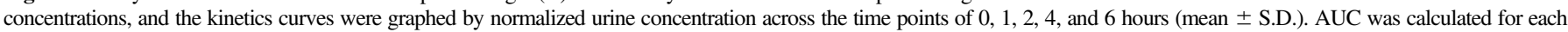

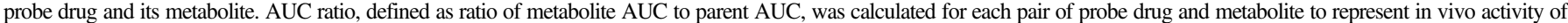

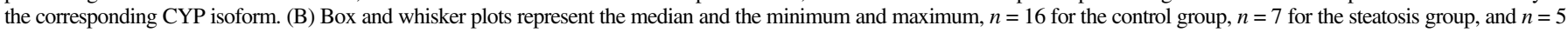

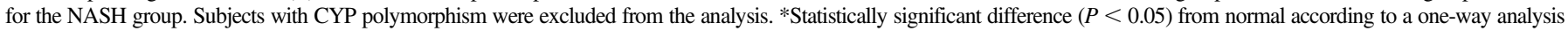
of variance Bonferroni's multiple comparison test. $P=0.8961$ for CYP1A2, $P=0.8876$ for CYP2C9, $P=0.4754$ for CYP2C19, and $P=0.7214$ for CYP3A4.

In conclusion, this study demonstrates a significant decrease in in vivo activity of CYP2C19 in adolescent NASH patients, whereas CYP1A2, CYP2C9, and CYP3A4 activity were unchanged, which is distinct from what was found in the ex vivo study in adults. These data indicate that adult and adolescents with NASH may need to be considered as unique patient populations in regards to CYP metabolism. Future studies on the pharmacokinetics of high-risk substrate drugs in larger research populations will be needed to translate these findings to guide actual 

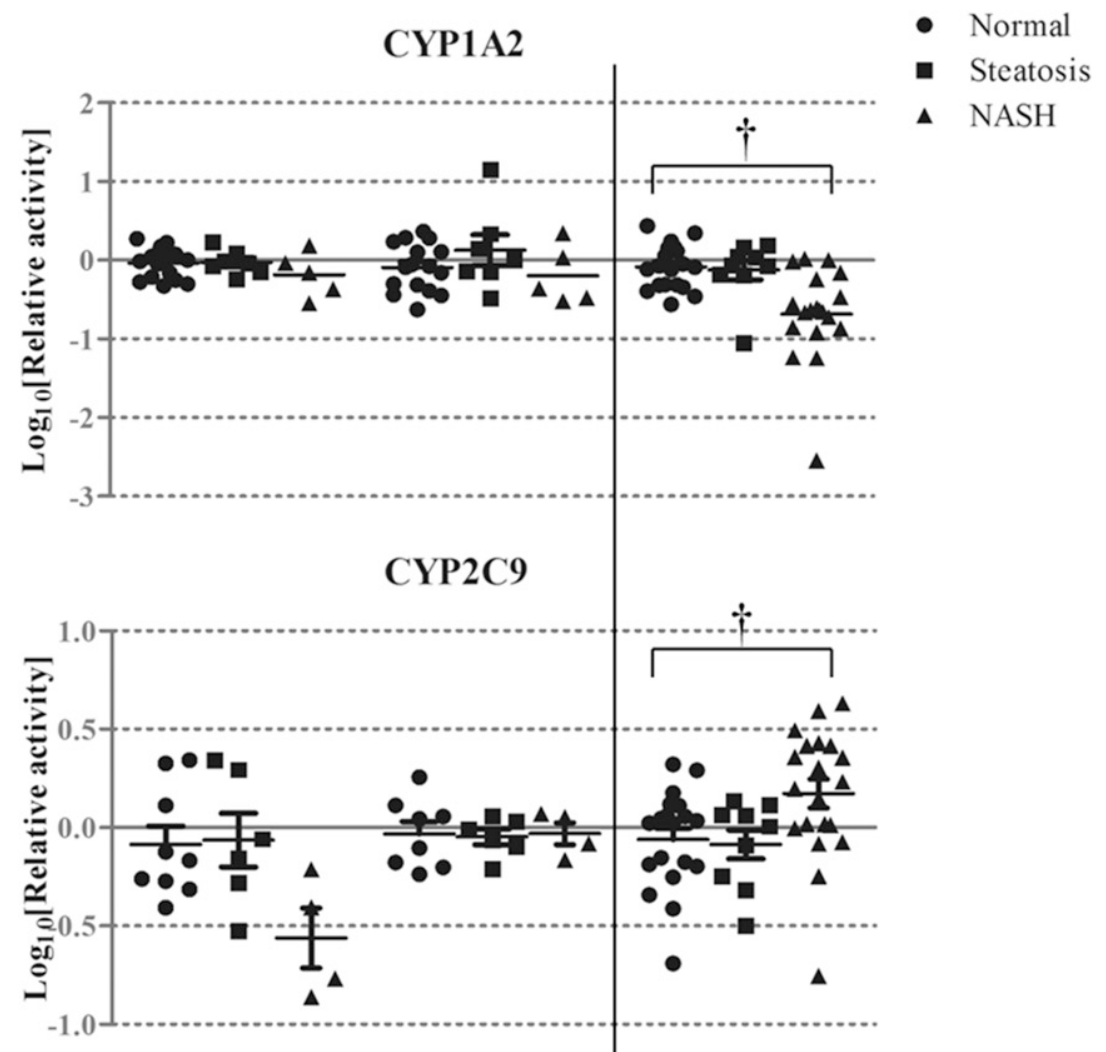

Fig. 3. Variance comparison of relative CYP activity Adult data were extracted from a previous ex vivo study, which was performed with human liver microsomes, while adolescent data were generated in the current in vivo study within an intact physiologic context. The relative enzymatic activities of NASH subjects were computed by normalizing to the mean activity of the control group. Data are presented in a logarithmic transformed scale. Each data point represents a measurement from one steatosis or NASH subject, and mean \pm S.E.M. is shown as well. † Statistically significant difference $(P<0.05)$ from normal within the group according to a one-way analysis of variance Bonferroni's multiple comparison test.

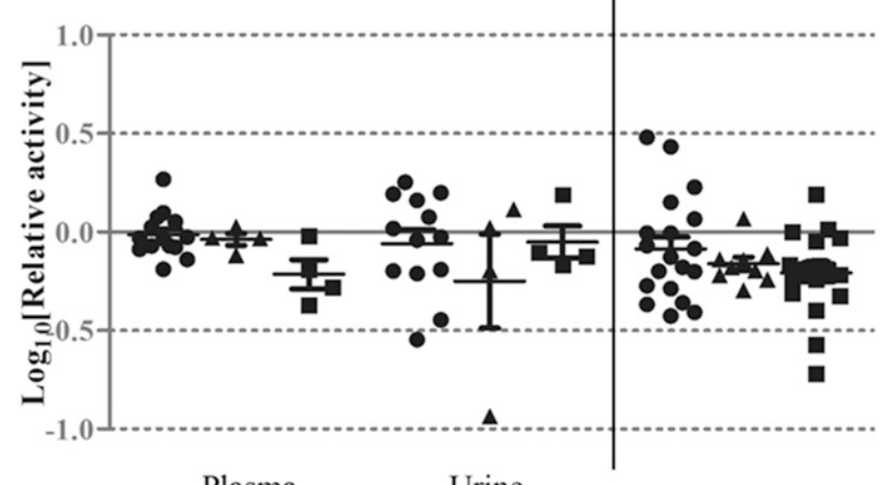

Plasma

Urine

Adolescent

Adult 
clinical practice. Ultimately, the possibility of NASH should be taken into consideration to adequately characterize the pharmacokinetics of CYP-metabolized drugs as well as make appropriate dose adjustments in pediatric clinical practice.

\section{Acknowledgments}

The authors thank Dr. Abrell Leif for assistance with the LC-MS/MS machine.

\section{Authorship Contributions}

Participated in research design: Canet, Xanthakos, Lavine, Erickson, Cherrington.

Conducted experiments: Li, Clarke, Erickson.

Performed data analysis: Li, Billheimer.

Wrote or contributed to the writing of the manuscript: Li, Canet, Clarke, Xanthakos, Lavine, Erickson, Cherrington.

\section{References}

Aitken AE and Morgan ET (2007) Gene-specific effects of inflammatory cytokines on cytochrome P450 2C, 2B6 and 3A4 mRNA levels in human hepatocytes. Drug Metab Dispos 35:1687-1693.

AlKhater SA (2015) Paediatric non-alcoholic fatty liver disease: an overview. Obes Rev 16: 393-405.

Anderson GD (2010) Developmental pharmacokinetics. Semin Pediatr Neurol 17:208-213.

Bambha K, Belt P, Abraham M, Wilson LA, Pabst M, Ferrell L, Unalp-Arida A, and Bass N: Nonalcoholic Steatohepatitis Clinical Research Network Research Group (2012) Ethnicity and nonalcoholic fatty liver disease. Hepatology 55:769-780.

Barshop NJ, Capparelli EV, Sirlin CB, Schwimmer JB, and Lavine JE (2011) Acetaminophen pharmacokinetics in children with nonalcoholic fatty liver disease. J Pediatr Gastroenterol Nutr 52:198-202.

Brill MJE, Diepstraten J, van Rongen A, van Kralingen S, van den Anker JN, and Knibbe CAJ (2012) Impact of obesity on drug metabolism and elimination in adults and children. Clin Pharmacokinet 51:277-304.

Canet MJ, Merrell MD, Hardwick RN, Bataille AM, Campion SN, Ferreira DW, Xanthakos SA, Manautou JE, A-Kader HH, Erickson RP, et al. (2015) Altered regulation of hepatic efflux transporters disrupts acetaminophen disposition in pediatric nonalcoholic steatohepatitis. Drug Metab Dispos 43:829-835.

Chalasani N, Younossi Z, Lavine JE, Diehl AM, Brunt EM, Cusi K, Charlton M, and Sanyal AJ (2012) The diagnosis and management of non-alcoholic fatty liver disease: practice Guideline by the American Association for the Study of Liver Diseases, American College of Gastroenterology, and the American Gastroenterological Association. Hepatology 55:2005-2023.

Chiney MS, Schwarzenberg SJ, and Johnson LA (2011) Altered xanthine oxidase and N-acetyltransferase activity in obese children. Br J Clin Pharmacol 72:109-115.

Clarke JD, Dzierlenga AL, Nelson NR, Li H, Werts S, Goedken MJ, and Cherrington NJ (2015) Mechanism of altered metformin distribution in nonalcoholic steatohepatitis. Diabetes 64 3305-3313

Clarke JD, Hardwick RN, Lake AD, Lickteig AJ, Goedken MJ, Klaassen CD, and Cherrington NJ (2014) Synergistic interaction between genetics and disease on pravastatin disposition. J Hepatol 61:139-147.

Crespo M, Lappe S, Feldstein AE, and Alkhouri N (2016) Similarities and differences between pediatric and adult nonalcoholic fatty liver disease. Metabolism 65:1161-1171

Cressman AM, Petrovic V, and Piquette-Miller M (2012) Inflammation-mediated changes in drug transporter expression/activity: implications for therapeutic drug response. Expert Rev Clin Pharmacol 5:69-89.

Dzierlenga AL, Clarke JD, Hargraves TL, Ainslie GR, Vanderah TW, Paine MF, and Cherrington NJ (2015) Mechanistic basis of altered morphine disposition in nonalcoholic steatohepatitis. $J$ Pharmacol Exp Ther 352:462-470.

Fisher CD, Lickteig AJ, Augustine LM, Ranger-Moore J, Jackson JP, Ferguson SS, and Cherrington NJ (2009) Hepatic cytochrome P450 enzyme alterations in humans with progressive stages of nonalcoholic fatty liver disease. Drug Metab Dispos 37:2087-2094.

Hardwick RN, Clarke JD, Lake AD, Canet MJ, Anumol T, Street SM, Merrell MD, Goedken MJ, Snyder SA, and Cherrington NJ (2014) Increased susceptibility to methotrexate-induced toxicity in nonalcoholic steatohepatitis. Toxicol Sci 142:45-55.

Hardwick RN, Ferreira DW, More VR, Lake AD, Lu Z, Manautou JE, Slitt AL, and Cherrington NJ (2013) Altered UDP-glucuronosyltransferase and sulfotransferase expression and function during progressive stages of human nonalcoholic fatty liver disease. Drug Metab Dispos 41 554-561.

Hardwick RN, Fisher CD, Canet MJ, Lake AD, and Cherrington NJ (2010) Diversity in antioxidant response enzymes in progressive stages of human nonalcoholic fatty liver disease. Drug Metab Dispos 38:2293-2301

Hardwick RN, Fisher CD, Canet MJ, Scheffer GL, and Cherrington NJ (2011) Variations in ATPbinding cassette transporter regulation during the progression of human nonalcoholic fatty liver disease. Drug Metab Dispos 39:2395-2402.
Hardwick RN, Fisher CD, Street SM, Canet MJ, and Cherrington NJ (2012) Molecular mechanism of altered ezetimibe disposition in nonalcoholic steatohepatitis. Drug Metab Dispos 40:450-460. Hines RN (2007) Ontogeny of human hepatic cytochromes P450. J Biochem Mol Toxicol 21 $169-175$.

Hisaka A, Ohno Y, Yamamoto T, and Suzuki H (2010) Prediction of pharmacokinetic drug-drug interaction caused by changes in cytochrome $\mathrm{P} 450$ activity using in vivo information. Pharmacol Ther 125:230-248.

Kearns GL, Abdel-Rahman SM, Alander SW, Blowey DL, Leeder JS, and Kauffman RE (2003) Developmental pharmacology-drug disposition, action, and therapy in infants and children. $N$ Engl J Med 349:1157-1167.

Kidd RS, Curry TB, Gallagher S, Edeki T, Blaisdell J, and Goldstein JA (2001) Identification of a null allele of CYP2C9 in an African-American exhibiting toxicity to phenytoin. Pharmacogenetics 11:803-808.

Kleiner DE and Makhlouf HR (2016) Histology of nonalcoholic fatty liver disease and nonalcoholic steatohepatitis in adults and children. Clin Liver Dis 20:293-312.

Lake AD, Novak P, Fisher CD, Jackson JP, Hardwick RN, Billheimer DD, Klimecki WT, and Cherrington NJ (2011) Analysis of global and absorption, distribution, metabolism, and elimination gene expression in the progressive stages of human nonalcoholic fatty liver disease. Drug Metab Dispos 39:1954-1960.

Loomba R, Sirlin CB, Schwimmer JB, and Lavine JE (2009) Advances in pediatric nonalcoholic fatty liver disease. Hepatology 50:1282-1293.

Magalhães J, Rodrigues AT, Roque F, Figueiras A, Falcão A, and Herdeiro MT (2015) Use of offlabel and unlicenced drugs in hospitalised paediatric patients: a systematic review. Eur J Clin Pharmacol 71:1-13.

Mason J, Pirmohamed M, and Nunn T (2012) Off-label and unlicensed medicine use and adverse drug reactions in children: a narrative review of the literature. Eur J Clin Pharmacol 68:21-28.

Matteoni CA, Younossi ZM, Gramlich T, Boparai N, Liu YC, and McCullough AJ (1999) Nonalcoholic fatty liver disease: a spectrum of clinical and pathological severity. Gastroenterology 116:1413-1419.

McGraw J and Waller D (2012) Cytochrome P450 variations in different ethnic populations. Expert Opin Drug Metab Toxicol 8:371-382.

Molleston JP, Schwimmer JB, Yates KP, Murray KF, Cummings OW, Lavine JE, Brunt EM, Scheimann AO, and Unalp-Arida A; NASH Clinical Research Network (2014) Histological abnormalities in children with nonalcoholic fatty liver disease and normal or mildly elevated alanine aminotransferase levels. J Pediatr 164:707-713.e3.

Molleston JP, White F, Teckman J, and Fitzgerald JF (2002) Obese children with steatohepatitis can develop cirrhosis in childhood. Am J Gastroenterol 97:2460-2462.

Morgan ET (1997) Regulation of cytochromes P450 during inflammation and infection. Drug Metab Rev 29:1129-1188.

Newton KP, Hou J, Crimmins NA, Lavine JE, Barlow SE, Xanthakos SA, Africa J, Behling C, Donithan M, Clark JM, et al.; Nonalcoholic Steatohepatitis Clinical Research Network (2016) Prevalence of prediabetes and type 2 diabetes in children with nonalcoholic fatty liver disease. JAMA Pediatr 170:e161971.

Rettie AE and Jones JP (2005) Clinical and toxicological relevance of CYP2C9: drug-drug interactions and pharmacogenetics. Annu Rev Pharmacol Toxicol 45:477-494.

Salem F, Johnson TN, Barter ZE, Leeder JS, and Rostami-Hodjegan A (2013) Age related changes in fractional elimination pathways for drugs: assessing the impact of variable ontogeny on metabolic drug-drug interactions. J Clin Pharmacol 53:857-865.

Schwimmer JB, Deutsch R, Kahen T, Lavine JE, Stanley C, and Behling C (2006) Prevalence of fatty liver in children and adolescents. Pediatrics 118:1388-1393.

Schwimmer JB, Deutsch R, Rauch JB, Behling C, Newbury R, and Lavine JE (2003) Obesity, insulin resistance, and other clinicopathological correlates of pediatric nonalcoholic fatty liver disease. J Pediatr 143:500-505.

Stojsavljević S, Gomerčić Palčić M, Virović Jukić L, Smirčić Duvnjak L, and Duvnjak M (2014) Adipokines and proinflammatory cytokines, the key mediators in the pathogenesis of nonalcoholic fatty liver disease. World J Gastroenterol 20:18070-18091.

Vet NJ, de Hoog M, Tibboel D, and de Wildt SN (2011) The effect of inflammation on drug metabolism: a focus on pediatrics. Drug Discov Today 16:435-442.

Yang J, Hao C, Yang D, Shi D, Song X, Luan X, Hu G, and Yan B (2010) Pregnane X receptor is required for interleukin-6-mediated down-regulation of cytochrome P450 3A4 in human hepatocytes. Toxicol Lett 197:219-226.

Zabalza M, Subirana I, Sala J, Lluis-Ganella C, Lucas G, Tomás M, Masiá R, Marrugat J, Brugada $\mathrm{R}$, and Elosua R (2012) Meta-analyses of the association between cytochrome CYP2C19 lossand gain-of-function polymorphisms and cardiovascular outcomes in patients with coronary artery disease treated with clopidogrel. Heart 98:100-108.

Zanger UM and Schwab M (2013) Cytochrome P450 enzymes in drug metabolism: regulation of gene expression, enzyme activities, and impact of genetic variation. Pharmacol Ther 138: 103-141.

Zhou Q, Yamamoto I, Fukuda T, Ohno M, Sumida A, and Azuma J (1999) CYP2C19 genotypes and omeprazole metabolism after single and repeated dosing when combined with clarithromycin. Eur J Clin Pharmacol 55:43-47.

Address correspondence to: Dr. Nathan J. Cherrington, University of Arizona, 1703 E. Mabel Street, Tucson, AZ 85721. E-mail: cherrington@pharmacy.arizona. edu 